\title{
Cool Color Map
}

National Cancer Institute

\section{Source}

National Cancer Institute. Cool Color Map. NCI Thesaurus. Code C85849.

A color map that varies from cyan at one extreme to magenta at the other extreme. 